\title{
A Literature Review on Teachers' Emotional Labor
}

\author{
Maolin Ye, Ye Chen \\ Management School, Jinan University, Guangzhou, China \\ Email: 290474046@qq.com
}

Received 13 October 2015; accepted 27 November 2015; published 30 November 2015

Copyright (C) 2015 by authors and Scientific Research Publishing Inc.

This work is licensed under the Creative Commons Attribution International License (CC BY). http://creativecommons.org/licenses/by/4.0/

(c) (i) Open Access

\begin{abstract}
In view of the teachers' professional characteristics, teachers are rated as high emotional workers. By reviewing the literatures of Chinese and foreign teachers' emotional labor research, this paper sums up four aspects about teachers' emotional labor: the definition of the concept of emotional labor, teacher's emotional display rules, teachers' emotional labor strategies and the antecedents and the consequences of teachers' emotional labor and also discusses the shortage of contemporary studies about teachers' emotional labor tentatively.
\end{abstract}

\section{Keywords}

Teachers, Emotional Labor, Emotional Display Rule, Emotional Labor Strategy

\section{Introduction}

As we know, teacher is not only a bridge between knowledge and students, but also a keeper of caring students' heart. Teaching is not only to teach accurately but also to show students the passion of teaching and interests of the teaching content. A teacher should have four qualities: having positive attention for students both inside and outside the classroom, communicating with parents of students patiently, getting along well with colleagues, and respecting the leaders. Even in personal life, teacher should also show more positive attitude towards life than any others. So what a teacher needs is not only good professional knowledge, but also better emotional management ability. Teacher is not only physical and mental laborer but also high emotional laborer. Therefore, it is very important to strengthen the research on teachers' emotional labor. By reviewing the literatures of Chinese and foreign teachers' emotional labor research, this paper sums up four aspects about teachers' emotional labor: the definition of the concept of emotional labor, teacher's emotional display rules, teachers' emotional labor strategies and the antecedents and the consequences of teachers' emotional labor. It also gives some suggestions about the study of teacher's emotional labor in future. 


\section{What Is Emotional Labor}

\subsection{The Definition of Emotional Labor}

The concept of emotional labor is presented to base on "emotion regulation". Hochschild pointed out that people will be based on their own scene to manage emotions and discussed why emotion is a kind of capital which can be used to exchange in 1979. In the study which Hochschild chose the flight attendants as the objects of research, Emotional labor is defined "For getting paid, the staff manage their emotions and performance to meet the requirements of visible facial expressions or body language which is required by organaization”, at the same time, three conditions of the occupation which involves "emotional labor" are also put forward: 1) There is face to face or voice direct dialogue; 2) it is under the supervision and control of the others; 3 ) in order to influence others 'emotion for the purpose (Hochschild, 1983).

Following Hochschild' definition of “emotional labor” concept, Ashforth \& Humphrey focused on the worker's observable behavior and considered emotional labor is "Staff performance proper emotional behavior according to organization requires” (Ashforth \& Humphrey, 1993). Morris \& Feldman considered that emotional labor contextually as social environments provoke different emotions, and that the emotional labor is "The staff express organizationally desired emotional behavior during interpersonal transactions, through the efforts, planning and control needed” (Morris \& Feldman, 1996). From the inner psychological activities perspective, Grandey (2000) defined emotional labor as "the process of regulating both feelings and expression for the organizational goals” (Grandey, 2000). And Diefendorff defined emotional labor as "the process of inner psychological activities in which staff monitor their own emotional expression and organizational requirements, and make efforts to reduce the gap” (Diefendorff, 2003). Some scholars think displaying and feeling of emotion is two important dimensions of emotional labor, the emotional labor of operationalization is defined as "showing real emotions, displaying pretended emotions and to suppress own mood and emotions” (Glomb \& Tews, 2004; Zhang et al., 2006).

We can found It is an important part of emotional labor through the above several common definition that the requirements of the organization, namely "emotional display rules”, and the individual strategy of emotional expression, namely, "emotional labor strategy”. In the work environment, "What kind of emotional behavior do they perform?" and "how to express the emotional behavior" are the focus of the study of emotional labor. In addition, according above definitions of emotional labor which have been given by many scholars from different angle, the emotional labor as a whole can be summed up in one sentence, emotional labor refer to that the individual express external emotional behavior of organizational requirements during interpersonal interaction, through feelings and adjustment of the inner emotions. Obviously, emotional labor is a multi-dimensional structure, the emotional laborer and interactive object are an integral part of the emotional labor. Emotional labor is affected by the interactive situation, social culture, the characteristics of the emotional labor and interactive objects, work characteristics, and other factors.

\subsection{The Relation between Emotional Labor and Emotional Regulation}

Emotional labor is put forward in the concept of emotional regulation. Scholar Gross discussed this set of concepts in discrimination points, considered that emotion regulation is "the process by which individuals influence which emotions they have, when they have them, and how they experience and express” (Gross, 1998). Elizabeth Levine Brown indicated that emotion regulation was based on self demand, and is not restricted by region and situation. Emotional labor occurs in the workplace, the individual in accordance with the organizational requirements of emotional expression make the appropriate adjustments emotion and express (Brown, 2011). Overall, 1) display rule of emotional labor is determined by the organization, and display rule of emotional regulation is determined by the individual; 2) purpose of emotional labor realizes the maximize benefits of the organization in order to obtain return of corresponding, and the purpose of emotion regulation is diverse and not only refers to a situation; 3) emotional labor generally refers to the emotional regulation and performance under the interpersonal interaction in the workplace, and the emotional regulation that can occur in the workplace and in the private life. Emotional labor belongs to the category of emotional regulation, is a special emotional regulation.

\section{Teachers' Emotional Display Rule and Strategy Research}

“Emotional Display Rules” of organizational requirements and “Emotional labor strategy” of individual using is 
an important part of emotional labor, in this part, the research status of the two part of teachers' emotional labor were sorted out.

\subsection{Emotional Display Rules}

Some scholars think identifying the emotional rules is essential to indicate whether teaching involves emotional labor (Zembylas, 2002). In fact, teachers need to follow the rules of emotional expression and management which was required by school in the work environment and even in the personal life. Winograd argues that the expression of the teachers' emotion should be done: 1) take care of students and show students enthusiasm; 2) express your great enthusiasm and passion for the teaching content in class; 3) try to avoid the display of extreme emotions in the teaching such as ultra mood very happy or angry sad; 4) to love their work; 5) to humorous to resolve their own errors and the students' errors (Winograd, 2003). Zembylas thought that teachers' emotional display rules can be summed up in one sentence, teacher should not express too strong and too weak emotions (Zembylas, 2005). Hongbiao Yin \& John Chi-Kin Lee point out the emotional display rules of Chinese teachers can be classified as four points: 1) Teaching should be full of passion; 2) hide negative emotions; 3) maintain a positive emotional state; 4) instrumentalise own emotions to achieve teaching goasl (Yin \& Lee, 2012).

Chinese scholar Chunying Liu proposed that teachers' emotional display rules was influenced by social cultural background and educational practices, was formulated on the basis of the organization's goal, and can help teachers to achieve the teaching goal, At the same time the scholar discusses the teachers should treat parents of students with (empathy, activeness, care, attentive listening, modesty), treat colleagues with (love and care, listening and responding appropriately, keeping a promise, respecting the privacy of others), treat the students with (sinceveness, accepting the students, maintaining the proper distance), treat school leaders with (respcting, maintaining authority, expressing their views and suggestions calmly), As well as the school advocate controlling negative emotions for teachers (Liu, 2012).

Through the above discussion it can be considered: 1) the rules of teachers' emotional expression not only exsit in class but also after class; 2) the rules of teachers' emotional expression for different interactive objects such as students, parents and leaders are different; 3) teacher's emotional display rules in the interest of the object of interaction requires teachers to restrain the expression of the negative emotions.

\subsection{Emotional Labor Strategies}

\subsubsection{Teachers' Emotional Strategies Category}

Hochschild thinks emotional labor has three strategies, namely, surface acting, deep acting and expression of naturally felt emotions. Surface acting is when the individual feeling is different from the emotion which is required by organaization, people don't adjust the inner feeling. They just perform required external emotional behavior. This is a kind of pretending emotion. Deep acting means that when individual feelings and organizational requirements emotions of expression are inconsistent, the employee changes internally felt emotions align with required emotional expressions of the organization. Expression of naturally felt emotions refers to the internal feelings of emotions and the organizational requirements for performance of emotional are consistent, employees reflect their emotions as they feel. When expression of naturally felt emotions was presented at the beginning, it was considered to be the true feelings of the show, don't need to work hard, and doesn't get much attention. Ashforth who emphasize the importance of emotional external behavior argues that although the expression of naturally felt emotions of the effort is not required, it is still one of emotional labor strategies (Ashforth \& Humphrey, 1993). Morris \& Feldman thought the expression of naturally felt emotions need to pay a little effort comparing with the other strategies (Morris \& Feldman, 1996). Later study confirmed that the expression of naturally felt emotions was indeed one of the emotional labor strategies (Diefendorff \& Croyle, 2005).

Zapf considered that "Deliberative Dissonance Action” was also a kind of emotional labor strategy, that is in some cases the requirements of emotional feeling are inconsistent with the requirements of emotional expression, for example, the organization require employee maintain inner neutral feeling and external positive behaviour (Zapf, 2002). Linfeng Yang (2008)' study found that deliberative dissonance action and expression of naturally felt emotions just pay less effort than surface acting and deep acting (Yang, 2008; Qian, 2012).

By reviewing the literatures, We can find that some scholars think emotional labor strategy have two factors, surface acting and deep acting, some scholars think emotional labor strategy consist of three factors, namely, 
surface acting, deep acting, and expression of naturally felt emotions, some scholars think emotional labor strategy have four sub dimensions, surface acting, deep acting, expression of naturally felt emotions and deliberative dissonance action. According to their own understanding of the emotional labor strategy, scholars choose one kind of emotional labor theory as the theoretical basis of their research. The majority adopt three factors. For example, Yanling Liu (2007) developed Teacher's emotional labor scale in Elementary and Middle school on the basis of the emotional labor theory of three factors (Liu, 2007). Research (Basim et al., 2013; Yang \& Yang, 2009) adopted surface acting, deep acting and expression of naturally felt emotions as emotional labor strategy. Lihua Miu (2009) found that elementary and middle school teachers' emotional labor includes four dimensions, deliberative dissonance dction, surface acting, deep acting and expression of naturally felt emotions, and developed the Scale of Teachers' Emotional Labor which has satisfying reliability and validity (Liu, 2009). Xueqin Zhang (2013) was only discusses surface acting and deep acting in the study of college English teachers' emotional labor (Zhang, 2013). Overall, the research mainly focused on the teachers of deep acting, surface acting and expression of naturally felt emotions, and showed a lack of research of deliberative dissonance action. At the same time, a lot of scholars pay more attention on the relation between emotional laborer(teachers) and emotional labor, a few scholars pay their attention on the relation between emotional labor and interactive objects (students, parents, and colleagues, etc.).

\subsubsection{The Usage of Teachers' Emotional Labor Strategy and Their Relationship}

Wenling Liu et al. (2013) and Xuehong Tian et al. (2009) adopted the theory of teacher's Emotional Labor Strategy which includes three dimension: surface acting, deep acting and expression of naturally felt emotions and found teachers use expression of naturally felt emotions more, follow by deep acting, surface acting least. (Liu et al., 2013; Tian \& Zhou, 2009). Lihua Miu (2009) found that elementary and middle school teachers' emotional labour includes four dimensions, deliberative dissonance action, surface acting, deep acting and expression of naturally felt emotions, and that the teachers use expression of naturally felt emotions and active deep action most, surface action least (Miu, 2009). Yuxian Xu (2012)' study was found the teachers use surface acting more than deliberative dissonance acting (Xu, 2012). Lijun Xiao (2012)' study found that the score of preschool teachers' emotional labor strategy from high to low respectively were expression of naturally felt emotions, surface acting and deep acting (Xiao, 2012).

Yanling Liu (2007)'s study found that A few teachers used only one kind of emotional labor strategy, most teachers often used two or more emotional labor strategies (Liu, 2007). Yanhua Hu et al. (2013) chose junior middle school teachers as research object and found that there were three kinds of teachers' emotional labor strategy combination: Low deep acting low expression of naturally felt emotions and High surface acting, The medium level of surface acting, deep acting and expression of naturally felt emotions, and High expression of naturally felt emotions High deep acting and Low surface acting group, and that the first strategy combination was the presence of the highest health risks for teacher, the latter of two health risk level was in turn reduce (Hu, 2013). In addition Xue-Hong Tian (2009) chose special education teachers as research object found that there are four kinds of emotional labor strategy combination, namely, the high expression of naturally felt emotions high deep acting low surface group, high expression of naturally felt emotions high surface high deep acting, low expression of naturally felt emotions low surface acting low deep acting group, and high deep acting high surface acting low expression of naturally felt emotions group. It can clearly be seen teachers' emotional labor strategies using to be with combination features (Tian, 2009).

Researching (Liu, 2007; Basim \& Begenirbaş, 2013; Mo, 2014)'s results show that expression of naturally felt emotions and surface acting are positively correlated to deep acting, surface acting, deep acting and expression of naturally felt emotions were significantly correlated with the total variables of emotional labor. It was inconsistent the conclusion of the relation between surface acting and expression of naturally felt emotions, such as no significant positive correlation, no significant negative correlation and significantly negative correlation in various research results (Liu, 2007; Basim et al., 2013; Mou, 2014).

Through the above research conclusions can be found: 1) The current can only determine the teacher will be preferred to use strategy of expression of naturally felt emotions, there are different conclusion for the order of priority among surface acting, deep acting and deliberative dissonance action. The priorty of the conditions and the impact factors, etc., need to be combined with the specific characteristics of the work to do further research; 2) there are the characteristics of combination for teachers to use the emotional labor strategy in the work life, at the same time there are different impact for teacher' health when using different combination; 3) we need to ex- 
plore whether there are certain regularity for the relationship among the different emotional labor strategies in the future.

\section{The Relationship between Teachers' Emotional Labor and Other Variables}

This is mainly involves the following two variables: one is the antecedent; other one is the consequences.

\subsection{There Are Many Influence Factors of Teachers' Emotional Labor, the Part of Several Common Important Antecedent to List Systematically}

\subsubsection{Personality Characteristics}

H. Nejat Basim et al. (2013) chose elementary and middle school teachers as research objects and found that big five personality traits can predict emotional labor strategy, surface acting was significantly predicted by neuroticism, deep acting was significantly predicted by openness to experience, openness to experience and agreeableness significantly predicted expression of naturally felt emotions (Basim \& Begenirbaş, 2013). Manyun Yang (2008) was based on Eysenck's personality theory, and explore the features of teacher's emotional labor and its relationship with personal mental health. The study showed emotional labor was positively correlated with psychoticism and negatively correlated with extraversion-introversion, but there was no significant correlation between teachers' emotional labor and neuroticism (Yang, 2003). Thus, personality characteristic is the important factor of emotional labor. These people whose extraversion, agreeableness and neuroticism features are prominent are more qualified for teaching.

\subsubsection{Emotional Intelligence}

Hong-biao Yin, John Chi-Kin Lee et al. (2013) chose elementary and middle school teachers as research objects and found the four first-order emotional intelligence factors had positive and significant correlations with two emotional labor strategies, namely, deep acting and expression of naturally felt emotion, but there were only weak, or even non-significant, correlations between surface acting and the four first-order EI factors (Yin et al., 2013). Wenkai Guo's (2014) study also got the above result. At the same time, as two sub dimensions of emotional intelligence, regulation of emotion and use of emotion are significant negatively correlated to surface acting. As two sub-dimensions of emotional intelligence, self-emotion appraisal and aothers' emotion appraisal are negatively correlated but not significantly to surface acting, But all dimensions of emotional intelligence are significant positively correlated to deep acting and expression of naturally felt emotions (Guo, 2014).

\subsubsection{Psychological Capital}

Ching-Sheue FU (2014)'s study used the four constructs of psychological capital, which include self-efficacy, optimism, hope, and resilience, to predict preschool teachers' emotional labor and indicate only self-efficacy could not be used to predict emotional labor, increasing preschool teachers' self-efficacy helps to reduce their emotional labor (Fu, 2014). Tuoyu Mo (2014)'s study found that primary and middle school teachers' interpersonal psychological capital have significant positive forecast effect to deep acting and expression of naturally felt emotions, and that have negative prediction function on surface acting, task-based psychological capital can positively predict expression of naturally felt emotions, deep acting and surface acting (Mou, 2014).

\subsubsection{Motivation}

Camelia Truta (2014) chose teachers as research objects and found intrinsic motivation is relevant only in relation to deep acting, while surface acting is not associated with any form of motivation. Intrinsic motivation is not itself a predictor of deep acting, it has predictive value only when associated with several organizational antecedents. At the same time, the scholar suggest a deeper analysis is required to identify the motives underlying employees' emotion management in the workplace (Truta, 2014). The relationship between internal and external motivation and emotional labor strategy should be further explored.

\subsubsection{Demographic Variables}

1) Seniority

Researches (Brown et al., 2014; Liu, 2007) found that there are positive correlation between seniority and teachers' deep acting, the teachers who work more longer are found to be most favorable to deep acting. Yang 
Sun (2013) found the preschool teachers were 11 year of school age, whose the expression of naturally felt emotions entered a rapid growth. With the increase of the school age, deep acting growed steadily. In the 6 - 10 year of school age group, deep acting reached a maximum value, subsequently declined. In the first 5 years of school age, surface acting showed a growth trend, and then began to fall. Except for less than 1 year of school age group, all the preschool teachers' emotional labor weren't balanced development. As a whole, the expression of naturally felt emotions developed more slowly than deep acting and surface acting. So we can see age impact teachers' emotional labor.

2) Gender

Yanling Liu (2007) chose primary and middle school teachers as the object of study found that there is significant gender differences in expression of naturally felt emotions, female teachers were higher than male, but the gender difference was not significant in the surface acting and deep acting (Liu. 2007). Xiaoning Chen (2010) chose colleges and universities young teachers as the object of study and found there are not significant gender differences in emotional labor which includes surface acting, deep acting and expression of naturally felt emotions but the surface acting of male is higher than that of the female, and the expression of naturally felt emotions of the female was significantly higher than that of male (Chen, 2010). Xuehong Tian et al. (2009)'s study found that there was not significant gender difference in the emotional labor of the special education teachers, but the surface acting and the deep acting of the female were higher than that of male, the female of emotional labor load was higher than that of male (Tian et al., 2009). So we can see that gender affect teachers' emotion work, but the role of gender may be different for different teachers groups.

3) Other

Xiaoning Chen et al. (2010)'s study found that the surface acting of unmarried individuals was significantly higher than that of married individuals, The expression of naturally felt emotions was significantly lower than that of the married individual (Chen, 2010). In addition, Yanling Liu (2007)'s study for primary and secondary school teachers found the expression of naturally felt emotions of unmarried individuals were significantly lower than that of married individuals, there was no significant difference in surface acting and deep acting between singles and married people. Lihua Mou (2009) chose primary and secondary school teachers as the object of study, and found whether a class sponsor have no significant main effect on Teachers' Emotional Labor (Mou, 2009), but Manyun Yang (2008)'s study found that the emotional labor burden of teachers who are in charge of a class is much higher than that of teachers who are not in charge of a class (Yang, 2008).

To sum up, there are many factors to affect emotional labor. These factors includes dynamic variables and static variables. There are numerous inconsistent conclusions in the present studies, which need to be further explored.

\subsection{The Consequences of Teacher's Emotional Labor Strategy}

Emotional labor has positive and negative effects, several common important consequences to be listed systematically in this part.

\subsubsection{Emotional Exhaustion}

Some studies found that surface acting had positive prediction function on emotional exhaustion, deep acting and expression of naturally felt emotions had negative prediction function on it (Basim, \& Begenirbaş, 2013; Zhang, 2013; Tian et al., 2009). Yang Sun’s Dynamic study found, the deep acting of the first test (T1) was significant negatively correlated with emotional exhaustion of six months later (T2) and one year later (T3). T2 deep acting was significant negatively correlated with T3 emotional exhaustion. T2 surface acting was significant positively correlated with T3 emotional exhaustion. T2 emotional exhaustion was significant positively correlated with T3 surface acting. There was the reciprocal relation between emotional labor and emotional exhaustion, We can infer they are relatively low the possibility of emotional exhaustion if teachers kept high-level of deep acting in the next year (Sun, 2013).

\subsubsection{Job Satisfaction}

Ling Yang and Mingjun Li (2009) confirmed surface acting were negatively correlated with job satisfaction, deep acting and expression of naturally felt emotions were positively correlated with job satisfaction. Emotional labor strategies are important factors for teacher's job satisfaction (Yang \& Li, 2009). Hongbiao Yin, et al. 
(2013) chose primary and secondary school teachers as the research object and found among the three emotional labor strategies, expression of naturally felt emotion, surface acting and deep acting, only expression of naturally felt emotion significantly influences teachers' sense of teaching satisfaction, whereas neither surface acting nor deep acting significantly predicts teaching satisfaction (Yin, 2013).

\subsubsection{Organizational Commitment}

Xinghua Ji (2009) chose junior and senior middle school teachers as the research object, explored the relationship between teachers' emotional labor and organizational commitment, the results showed that there was a positive correlation between deep acting and organizational commitment, surface acting are not related to organizationa commitment, expression of naturally felt emotions and ideal commitment were positive correlation, expression of naturally felt emotions are not relate to affective commitment and normative commitmen. Deep acting can predict affective commitment, normative commitment and investment commitments (Ji, 2009).

\subsubsection{Other}

Wenling Liu, et al. (2013) chose primary and secondary school teachers as research object and found expression of naturally felt emotions and deep acting are positively correlated to teachers' vocational well-being, surface acting are negatively correlated to teachers’ vocational well-being but no significant (Liu et al., 2013). Manyun Yang's study (2008) found teacher's emotional labor was significantly correlated with its symptoms, the higher the teacher' burden of emotional labor, the higher the scores of psychological symptoms, the relationships between scl-90 and three dimensions of emotional labor: variety of expressed emotions, deep acting and customer interaction show significant positive correlation, and requirement to emotional display, surface acting has not significant correlation. Lijun Xiao's study found that teacher's emotional labor was significantly correlated to job performance, surface acting was negatively correlated to job performance, deep acting and expression of naturally felt emotions were positively correlated to job performance (Xiao, 2012).

From the above discussion can be found that teachers' emotional labor is not only has positive effect but also has negative effect, Its negative effects is mainly derived from the use of surface behavior strategies, according to the theory of conservation of resources, strategy of surface acting is large amount of resource attenuation than deep acting and expression of naturally felt emotions, that is also not conducive to the timely supply of resources. This seems particularly important that how to reduce teachers' surface acting, increasing teachers' expression of naturally felt emotions and deep acting, promoting the sustainable development of teachers' emotional resources and mitigation of job burnout from teacher's point of view.

\section{Conclusions}

Teachers' emotional labor study can be found. Firstly, scholars pay more attention to emotional labor between teachers and students, ignoring emotional labor between teachers and parents of students, emotional labor between teachers and colleagues, emotional labor between teachers and leaders. Empirical research object of teacher' emotional labor is more limited to basic education field; it is lack of technical education teachers, special education teachers and online teachers to explore. At the same time, the research mainly focuses on the emotional laborer themselves, lacks the research on the influence of the characteristics of the interactive objects, and also lacks the research of the relation between the characteristics of interaction objects (students, colleagues, parents and leader, etc.) and teacher's emotional labor. Secondly, emotional labor strategy is the core of emotional labor. Currently the study mainly contains the antecedents and the consequences of surface acting, deep acting and expression of naturally felt emotions. The empirical research of deliberative dissonance action still needs to be strengthened. Again, the teacher's emotional labor strategy exists in combination, and the current research on the emotional labor strategy is mainly based on a single strategy as the research object, the lack of a combination of strategies as the object of research. In addition, the present study shows that the teacher who has an extroversive personality may do more expression of naturally felt emotions and deep acting, so according to the demand of job characteristics, we should test personality of candidate for teacher. Emotional intelligence is an important factor of emotional labor; school should increase the emotional intelligence of teachers through the training of teachers. Finally, some conclusions of the empirical study are not consistent, we need to research further.

Education is the foundation of a country to be rich and powerful. The teacher is the most important part of education system, teacher's emotion is an educational tool directly affecting the students' physical and mental 
development and learning, teachers' emotional labor directly influences teachers’ work efficiency and quality, and it is multifaceted to the factors of influencing teachers' emotional labor; therefore in the succession to previous studies, we should do well on the transverse study and also don't ignore the longitudinal transverse comparison study.

\section{References}

Ashforth, B. E., \& Humphrey, R. H. (1993). Emotional Labour in Service Roles: The Influence of Identity. Academy of Management Review, 18, 88-115.

Basim, H. N., Begenirbaş, M., \& Yalçin, R. C. (2013). Effects of Teacher Personalities on Emotional Exhaustion: Mediating Role of Emotional Labor. Educational Consultancy and Research Center, 13, 1488-1496.

Brown, E. L. (2011). Emotion Matters: Exploring the Emotional Labor of Teaching. Ph.D. Tesis, Pittsburgh: University of Pittsburgh.

Brown, E. L., Horner, C. G., Kerr, M. M., \& Scanlon, C. L. (2014). United States Teachers’ Emotional Labor and Professional Identities. Kedi Journal of Educational Policy, 11, 205-225.

http://www.researchgate.net/publication/271136874

Chen, X. N. (2010). The Empirical Study of Yong Univerity Teachers’ Emotional Labor. Heilongjiang Researches on Higher Education, 12, 23-26.

Ching-Sheue, F. U. (2014). An Exploration of the Relationship between Psychological Capital and the Emotional Labor of Taiwanese Preschool Teachers. Journal of Studies in Social Sciences, 7, 226-246.

Diefendorff, J. M., \& Gosserand, R.H. (2003). Understanding the Emotional Labor Process: A Control Theory Perspective. Journal of Organizational Behavior, 24, 945-959. http://dx.doi.org/10.1002/job.230

Diefendorff, J. M., Croyle, M. H., \& Gosserand, R. H. (2005). The Dimensionality and Antecedents of Emotional Labor Strategies. Journal of Vocational Behavior, 66, 339-357. http://dx.doi.org/10.1016/j.jvb.2004.02.001

Glomb, T. M., \& Tews, M. J. (2004). Emotional Labor: A Conceptualization and Scale Development. Journal of Vocational Behavior, 64, 1-23. http://dx.doi.org/10.1016/S0001-8791(03)00038-1

Grandey, A. A. (2000). Emotion Regulation in the Workplace: A New Way to Conceptualize Emotional. Journal of Occupational Health, 5, 95-110. http://dx.doi.org/10.1037/1076-8998.5.1.95

Gross, J. J. (1998). The Emerging Field of Emotion Regulation: An Integrative Review. Review of General Psychology, 2, 271-299. http://dx.doi.org/10.1037/1089-2680.2.3.271

Guo, W. K. (2014). The Relationship between College Teachers’ Emotional Work Policy, Emotional Intelligence and Job Burnout. The Guide of Science \& Education, 11, 76-79.

Hochschild, A. R. (1983). The Managed Heart: Commercialization of Human Feeling. Berkeley, CA: University of California Press.

Hu, Y. H., Cao, X. M., \& Luo, Z. H. (2013). What Is Composite Mode of Junior Middle School Teachers' the Emotional Labor Strategy and How to Manage teachers. Modern Primary and Secondary Education, 4, 81-84.

Ji, X. H. (2009). The Study of the Relationship between Emotional Labor and Organization Commitment for Teachers. Journal of Huaibei Professional and Technical College, 1, 91-93.

Li, Q. (2012). Review of Emotional Labor Research at Home and Broad. Journal of postgraduate of Zhongnan University of Economics and Law, 3, 102-107.

Liu, L. H. (2009). Research on Emotional Labor of Elementary and Middle School Teachers. Master's Thesis, Chongqing: Chongqing University.

Liu, W. L., Chen, R., Lou, X. M., Liu, X., \& Liu, Y. L. (2013). Relationship between Primary and Middle School Teachers’ Emotional Work Strategies and Occupational Well-Being: On Moderating Effects of Psychological Capital. Journal of Southwest China Normal University: Natural Science Edition, 38, 152-157.

Liu, Y. C. (2012). Discussion on Teachers’ Emotional Display Rules. Journal of Zhejiang Normal Uiversity: Social Sciences, 63, 6-11.

Liu, Y. L. (2007). Research on Teacher's Emotion Work in Elementary and Middle School. Ph.D. Thesis, Chongqing: Southwest University.

Morris, J. A., \& Feldman, D. C. (1996). The Dimensions, Antecedents and Consequences of Emotional Labor. Academy of Management Review, 21, 986-1010.

Mou, T. Y. (2014). The Research on Relationship among Primary and Secondary School Teachers' Psychological Capital, Emotion Labor Strategies and Job burnout. Master’s Thesis, Changsha: Hunan Normal University. 
Sun, Y. (2013). The Developmental Characteristics of Preschool Teachers' Emotional Labor and Its Relation to Emotional Exhaustion. Ph.D. Thesis, Changchun: Northeast Normal University.

Tian, X. H., Zhou, H. Y., \& Chen, D. W. (2009). A Survey on Emotional Labor of Special Education Teachers. Chinese Journal of Special Education (Monthly), 8, 50-56.

Truta, C. (2014). Emotional Labor and Motivation in Teachers. Procedia - Social and Behavioral Sciences, 127, $791-795$. http://dx.doi.org/10.1016/j.sbspro.2014.03.356

Winograd, K. (2003). The Functions of Teacher Emotions: The Good, the Bad, and the Ugly. Teachers College Record, 105, 1641-1673. http://dx.doi.org/10.1046/j.1467-9620.2003.00304.x

Xu, Y. X. (2012). Studies on Emotional Labor Strategy of Secondary School Teachers and Its Relationship with Teaching Efficacy and Job Burnout. Master's Thesis, Wuhan: Hubei University.

Yang, L. F., Yu, X. N., \& Fan, T. W. (2008). The Dimension of Emotional Labor Structure Research. Journal of Inner Mongolia Agricultural University: Social Science Edition, 10, 262-265.

Yang, L., \& Li, M. J. (2009). The Relationship among Emotion Work Strategies, Characters and Job Satisfaction in Primary and Middle School Teachers. Psychological Development and Education, 3, 89-100.

Yang, M. Y. (2008). The Study on the Emotional Work of Elementary and Middle School Teacher and It's Relation with Traits and Mental Health. Master's Thesis, Chongqing: Southwest University.

Yin, H. B., \& Lee, J. C.-K. (2012). Be Passionate, But Be Rational as Well: Emotional Rules for Chinese Teachers’ Work. Teaching and Teacher Education, 28, 56-65. http://dx.doi.org/10.1016/j.tate.2011.08.005

Yin, H.-B., Lee, J. C.-K., Jin, Y.-L., \& Zhang, Z.-H. (2013). Exploring the Relationship among Teachers’ Emotional Intelligence, Emotional Labor Strategies and Teaching Satisfaction. Teaching and Teacher Education, 35, 137-145. http://dx.doi.org/10.1016/j.tate.2013.06.006

Zapf, D. (2002) Emotion Work and Psychological Well Being: A Review of the Literature and Some Conceptual Considerations. Human Resource Management Review, 12, 237-268. http://dx.doi.org/10.1016/S1053-4822(02)00048-7

Zembylas, M. (2002). Structures of Feeling in Curriculum and Teaching: Theorizing the Motional Rules. Educational Theory, 52, 187-208. http://dx.doi.org/10.1111/j.1741-5446.2002.00187.x

Zembylas, M. (2005). Discursive Practices, Genealogies, and Emotional Rules: A Poststructuralist View on Emotion and Identity in Teaching. Teaching and Teacher Education, 21, 935-948. http://dx.doi.org/10.1016/j.tate.2005.06.005

Zhang, H. H., Ling, W. Q., \& Fang, L. L. (2006). A Review on Emotion Work. Advances in Psychological Science, 14, 111119.

Zhang, X. Q. (2013). To Analyze Emotional Labor of University Teachers. Journal of TaiYuan Urban Vocational College, 9 , 120-122. 